\title{
ANALYSIS OF COMPLEX VARIABLE PROCESSES OF ELASTIC-PLASTIC DEFORMATION BY MEANS OF THE HIERARCHICAL CONSISTENCY OF MATERIAL MODELS
}

\author{
Boris Melnikov ${ }^{1}$, Artem Semenov ${ }^{2}$ \\ ${ }^{l}$ Department of Strength of Materials, Institute of Civil Engineering, Saint-Petersburg State Polytechnical University, \\ Polytechnicheskaya Str. 29, 195251 Saint-Petersburg, Russia. \\ ${ }^{2}$ Department of Mechanics and Process Control, Institute of Applied Mathematics and Mechanics, Saint-Petersburg \\ State Polytechnical University, Polytechnicheskaya Str. 29, 195251 Saint-Petersburg, Russia.
}

E-mail: ${ }^{1}$ kafedra@ksm.spbstu.ru (corresponding author); ${ }^{2}$ semenov.artem@googlemail.com

\begin{abstract}
A multimodel analysis of the elastic-plastic behavior of materials and structural components under complex non-proportional variable loading is considered. The multimodel elastic-plastic analysis is based on the developed library of elastic-plastic models and a system of criteria allowing the selection of adequate models. A unified form of material model description is given intended for the efficient finite element implementation. Efficiency of the multimodel analysis is illustrated considering several examples.
\end{abstract}

Keywords: plasticity, complex loading, multimodel analysis, finite element computations.

\section{Introduction}

The improvement of elastic-plastic constitutive equations is stimulated by continuously increasing requirements for the material economy and structure reliability with the simultaneous complication of loading programs and an increase of mechanical loads and operating temperatures. This has led to the necessity of the experimental investigation and the phenomenological modeling of complex phenomena of irreversible deformation processes. Over the past few decades many experimental and theoretical studies have been devoted to the development elastic-plastic constitutive equations, in order to better predict the inelastic material behavior under the combined variable non-proportional loading. The number of developed models increases from year to year. The present situation was reviewed by the number of authors (Khan, Huang 1995), (Jirasek, Bazant 2001), (Kafka 2001), (ed. Lemaitre 2001), (Zyczkowski 1981), (Vasin 1990), for concrete structures (Benin et al. 2013) and for soils (Kalinin 2013).

At present, an unified universal theory of elasto-plastic deformation is absent. Unfortunately even the most accomplished theories cannot provide always an acceptable accuracy of the deformation process description for an arbitrary structure under a wide range of loadings. The hierarchical multimodel and multilevel stress analysis can be considered as one of the most effective approaches to the numerical simulation of the inelastic behavior of complex structures. Using different elastic-plastic models for different structure approximation levels can reduce the total computation time without an accuracy loss. Such method of adaptive constitutive modeling is based on the using hierarchical sequence of elastic-plastic models for different structure approximation levels (body, element, point).

\section{Basical principles of multimodel analysis}

It was claimed (Prager 1949), (Novozhilov 1964), (Kolarov et al. 1979) that in the general case it is impossible to use one-model approach to elastic-plastic analysis of deformation processes.

In nowadays conditions of absent of universal elastoplasticity theory, which is applicable to arbitrary paths of loading, the multimodel approach (Melnikov, Semenov 1995), (Semenov, Melnikov 1998) to the stress analysis of inelastic behavior of structures under complex nonproportional loading is probably the most rational.

The main features of the developed multimodel approach (Melnikov, Semenov 1995) are the following:

- certification of the theories of elasto-plasticity in order to define the their application area and the possibility to describe special effects;

- creation of the library of elasto-plastic models;

- determination of the selection criteria system;

- carrying out multi-variant sequential clarifying computations.

In the finite element (FE) computations the selection of the adequate material model is performed iteratively with using different models from the hierarchical sequence. Iteration control is carried out in accordance with requirements to the accuracy and the availability of the additional experimental data.

\section{Characteristic effects of elastic-plastic behavior}

The used in FE simulations the automatic selection of elastic-plastic models is based on the classification of effects (phenomena) of inelastic behavior. Elastic-plastic deformation of metallic materials under variable uniaxial and multiaxial loading shows the existence of various phenomena, which as a rule are not described by many material models. The systematic analysis of elastic-plastic effects is performed in (Benallal et al.1985), (Tanaka et al. 1985), (Getsov et al. 2002), (Semenov et al. 2005), (Semenov et al. 2007), (Getsov et al. 2009) for monotonous and cyclic processes, proportional and non-proportional paths of loading, rate-dependent and rate-independent behavior, reversible and irreversible deformation processes. Some characteristic phenomena under non-proportional loading are presented in Table 1. 
Table 1. Some characteristic phenomena of elastic-plastic behavior of metallic materials

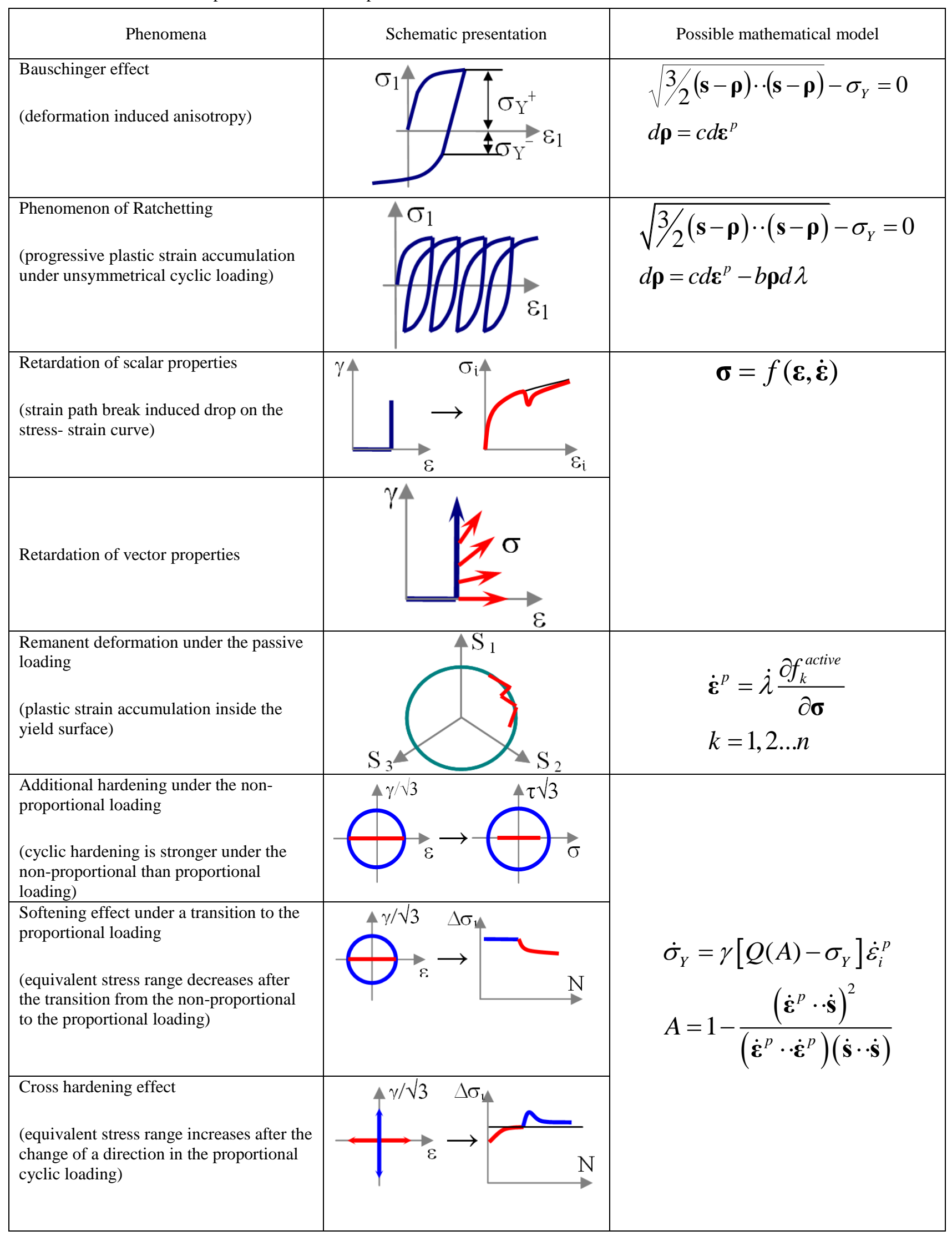


Table 1. (continued)

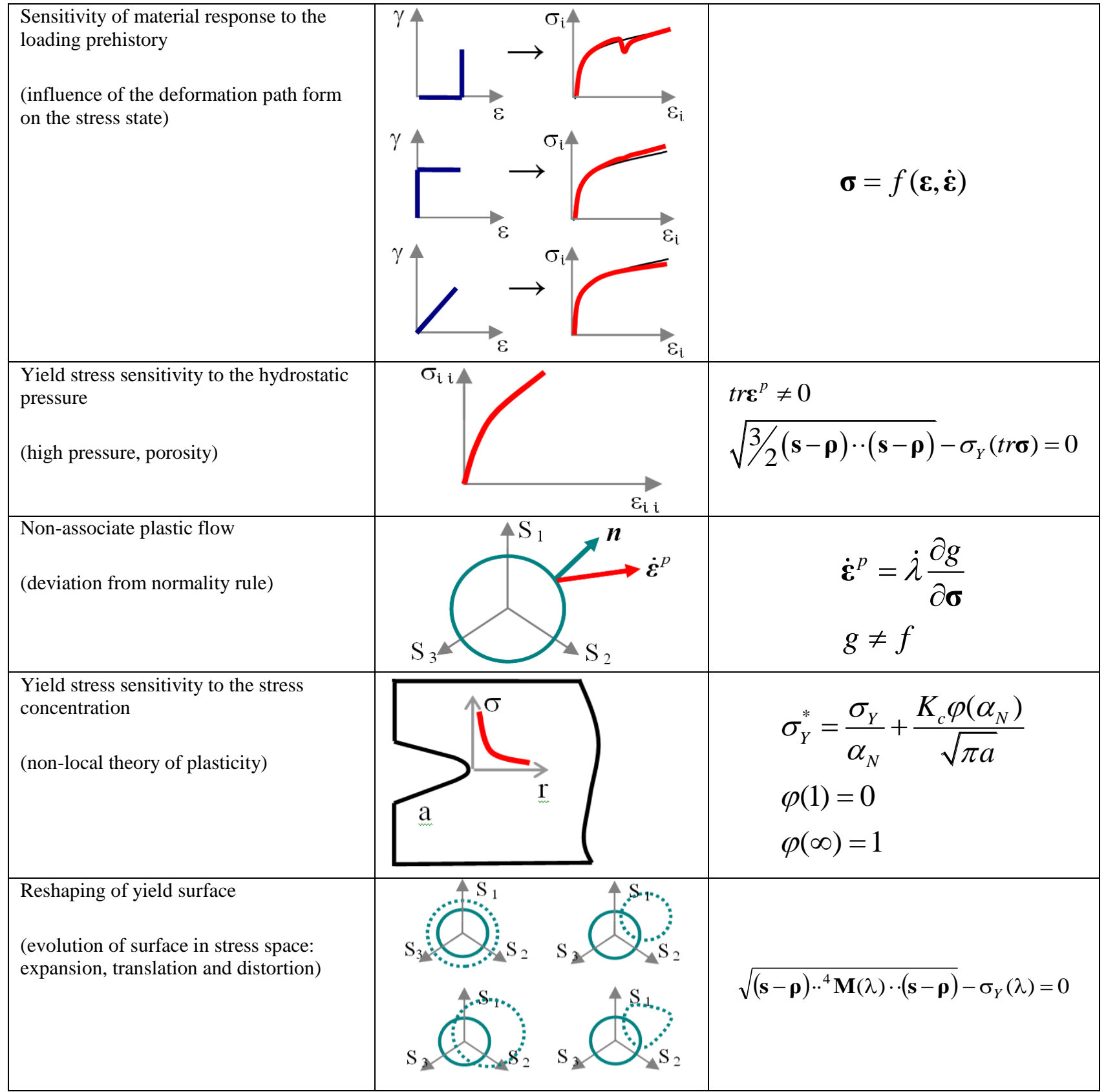

The notations are introduces in Table 1: $\varepsilon$ is the strain tensor; $\sigma$ and $s$ are the stress tensor and its deviator; $\rho$ is the micro-stress tensor deviator, characterizing a location of yield surface center; $\dot{\boldsymbol{\varepsilon}}^{p}$ denotes the tensor of plastic strain rates; $\sigma_{Y}$ is the yield stress; $\dot{\boldsymbol{\varepsilon}}_{i}^{p}=\sqrt{\frac{2}{3} \boldsymbol{\varepsilon}^{p} \cdot \boldsymbol{\varepsilon}^{p}}$ is the equivalent (von Mises) plastic strain rates; $\lambda$ is the Odquist's parameter (path arclength in the space of plastic strains), in particular for von Mises criterion $\lambda=\int \sqrt{\frac{2}{3} \dot{\boldsymbol{\varepsilon}}^{p} \cdot \dot{\boldsymbol{\varepsilon}}^{p}} d t ; g$ is the plastic potential function; $f$ is the yield surface function; $A$ - non-proportionality parameter; $\mathrm{n}$ is normal to the yield surface; $\alpha_{N}$ is Neuber's stress concentration coefficient; $K_{c}$ is the fracture toughness; $a$ is a crack length; the operator .. denotes a double contraction (a double scalar product of tensors), so $\mathbf{A} \cdot \mathbf{B}=A_{i j} B_{j i} \quad(i, j=1,2,3) ; c$ and $b$ are constants of material. 


\section{Library of elasto-plastic models}

The created for the multimodel numerical analysis library of elasto-plastic models represents a generalized data base, containing an information about restrictions on the area of model applications, list of basic experiments for material parameters measurement, continual mathematical model of deformation process, discrete numerical model, algorithm of computations, implementation into finite element program, recommendations about optimal computation strategy.

At present the developed library of rate-independent (elasto-plastic) models, which is implemented into finite element program PANTOCRATOR, contains:

- Plastic flow theory with different isotropic-kinemati hardening rules (constitutive equations of these "classical" models belong to linear tensorial equations convenient for computations).

- Rheologic (structural) models (they possess thermodynamic foundation, obvious creation and improvement procedures).

- Multisurface theory with one active surface (the model is developed with aim to describe the processes of passive non-proportional loading with a high accuracy).

- Endochronic theory of plasticity (the model does not consider a yield surface concept and uses the same relations for the loading and unloading). Detailed description of this theory is considered in (Kadashevich, Pomytkin 2013).

\section{Unified description of the constitutive equations}

The uniform mathematical description of elastic-plastic constitutive equations is topical in multimodel analysis for the creation of the library of material models. It provides simplification of program realization and comparison of model possibilities. A powerful tools for the formulation of elastic-plastic constitutive equations gives the irreversible thermodynamic approach with using internal state variables. All considered in the present investigation the elasto-pleastic models are rewritten in the unified, quite general mathematical form. The rate-independent functional-type constitutive equations are used in the general case of elsto-plastic material (Semenov 1996). These very complex and inconvenient for computation equations can be simplified in the extended case of quasi-linear differential evolution equations and can be presented as quasi-linear differential tensorial constitutive equation (Semenov 1996):

$$
\dot{\boldsymbol{\sigma}}={ }^{4} \boldsymbol{D}^{e p}\left(\boldsymbol{\varepsilon}, \boldsymbol{\sigma}, \boldsymbol{\chi}^{(k)}, T\right) \cdot \dot{\boldsymbol{\varepsilon}}+\boldsymbol{R}^{e p}\left(\boldsymbol{\varepsilon}, \boldsymbol{\sigma}, \chi^{(k)}, T\right) \dot{T}
$$

where $\boldsymbol{\varepsilon}$ and $\boldsymbol{\sigma}$ denote strain and stress tensors; $\chi^{(k)}$ are internal state variables $(k=1,2, \ldots, n)$ for the description of the dissipative microstructural processes; $T$ is temperature; ${ }^{4} \boldsymbol{D}^{e p}$ and $\boldsymbol{R}^{e p}$ are a fourth-rank tensor of elasto-plastic moduli and second-rank tensor, describing material response to the change of temperature.

The introduced internal state variables $\boldsymbol{\chi}^{(k)}$ usually represent second-rank tensors or scalars. The evolution equations for the internal state variables are defined by the first-order differential relations:

$$
\dot{\chi}^{(k)}=\dot{\lambda} \mathbf{b}^{(k)}\left(\boldsymbol{\varepsilon}, \boldsymbol{\sigma}, \boldsymbol{\chi}^{(i)}, T\right),
$$

where the multiplier $\dot{\lambda}$ is defined on the base of the consistency plastic conditions for rate-independent models or from the uniaxial creep curves for rate-dependent material models.

\section{Selection criteria of adequate material model}

One of the central problems in the multimodel approach is a formulation of the selection criteria system, which is directly based on the classification of elastoplastic models and their applicability areas. The system of selection criteria offers the necessary and sufficient conditions for material model including an information about allowable loading conditions, available experimental data. The selection of the suitable material model, which is the simplest among models satisfying the necessary conditions, may be carried out by means of fulfillment of sequential computational experiments.

The classical examples of selection criteria are comparison of plastic strain level with elastic strain level (leading to selection between Levy-Mises or PrandtlReuss models) and curvature and twist of the loading trajectory. In addition to the above the new selection criterion for structures with inhomogeneous stress states is formulated on the base of results of numerous computational experiments with elasto-plastic structures of different degree of complexity. The proposed selection criterion concerns consideration of geometrical regulated levels of plastic deformation analysis. Similarly to (Melnikov, Semenov 1995), (Melnikov, Semenov 1996), (Semenov, Melnikov 1998), (Semenov 1996), (Zyczkowski 1981) the following level hierarchy is considered:

- Body level B corresponds to the body or complex structure being object of simulation as a whole. "Integral" stress analysis is carried out for the original boundary value problem. Zones of plasticity have local character in most cases.

- Element level $\mathbf{E}$ corresponds to a part of the structure, domain with a possible defect, a representative volume element, a superelement or an individual finite element. "Semi-integral" stress analysis is performed in this case. Zones of plasticity have extensive character in most cases.

- Point level $\mathbf{P}$ corresponds to the primary level, relating to selected points of structure or to elements with homogeneous strain-stress state. "Local" stress analysis is carried out for simplest geometrical object. The whole object is a zone of plasticity in this case.

The criterion is in the approval: a complexity of the applying theory of plasticity have to correspond to the level of the structure approximation. The levels $\mathbf{E}$ and $\mathbf{P}$ with more detailed description of the structure geometry and with the possibility of extensive zones of plasticity require the using more complex variants of the plasticity theory, adequate to the complexity of loading process. 
Using quite simple models is sufficient for level B, whereas intensive deformation in the local zones of plasticity is smoothed by the extensive elastic domain.

\section{Results of multimodel finite element analysis}

A wide range of inelastic material models, including mentioned above, has been implemented into the finite element program system PANTOCRATOR (Semenov 2003), (FE program PANTOCRATOR 2013), which is especially designed for the simulation of solids and structures with complex mechanical properties.

A comparison of the results of finite element simulations and experimental data for the problems concerning structures corresponding to the level B (various frames, pipelines, antennas, vapor producing plant, ship gearbox housing, gas generator and vessel of nuclear reactor) demonstrates relative proximity of different theories results. However, the numerous finite element computations corresponding to the level $\mathbf{E}$ of the structures (fragment of rolling mill, fastenings of vapor producing plant, various fastening knots, socket and circular ring) have demonstrated that the considerable differences in stress-strain states take place for different theories of plasticity under conditions for the extended zone of plasticity and complex history of loading. The computations corresponding to the level $\mathbf{P}$ were carried out on tubular specimens of $1 \mathrm{X} 18 \mathrm{H} 10 \mathrm{~T}$ steel under various combined cyclic loadings, including polygonal and circular paths of deformation. The predictions of different theories for the problems corresponding to level $\mathbf{P}$ can differ greatly and qualitatively.

The comparison of results of multimodel analysis corresponding to the level $\mathbf{P}$ with experimental data are given in Fig. 1-2. Detailed description of problems can be found in (Semenov 1996), (Izotov et al. 2001). The example of multimodel analysis corresponding to the level $\mathbf{E}$ for thin circular aluminum ring being the part of a more complex structure is shown in Fig. 3. The strainstress analysis of the vapor producing plant (Fig. 4a) is considered here as an example corresponding to level $\mathbf{B}$. The impact loading in the horizontal direction is defined in finite element computations by accelerations of the fixture points.

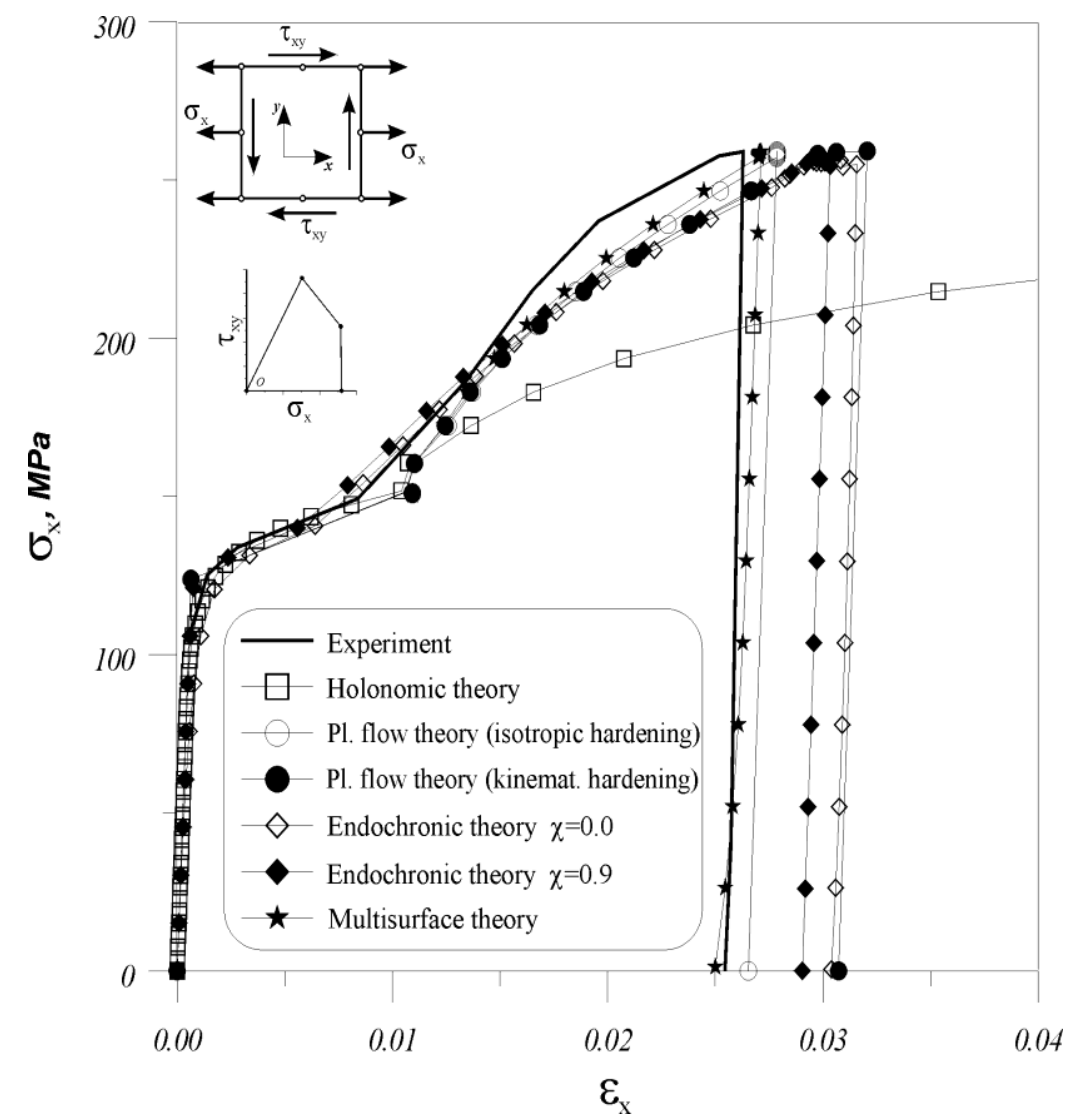

Fig. 1. Comparison of different model predictions with experimental results for thin-walled tubular specimen under combined axial tension-torsion (Ottosen 1979), (Szabo 1984), (Semenov 1996). 


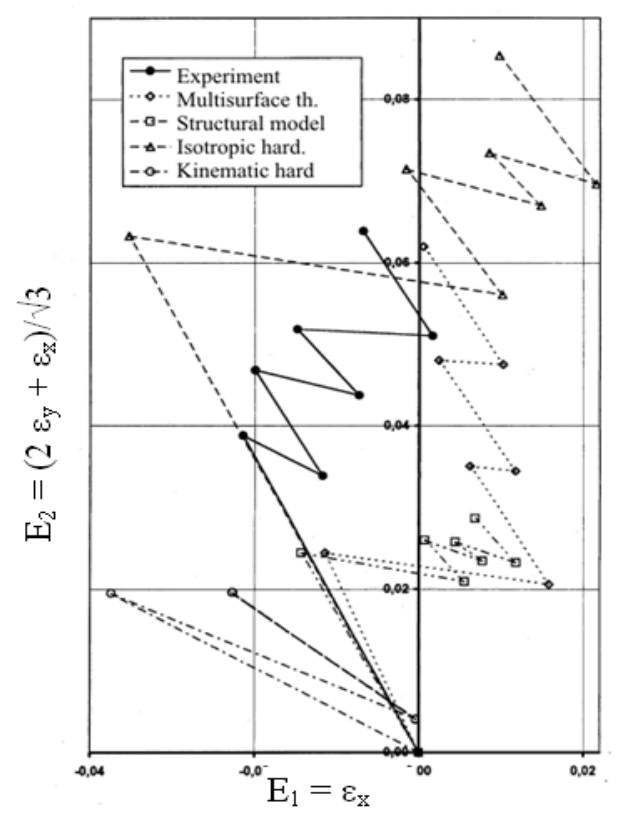

a)

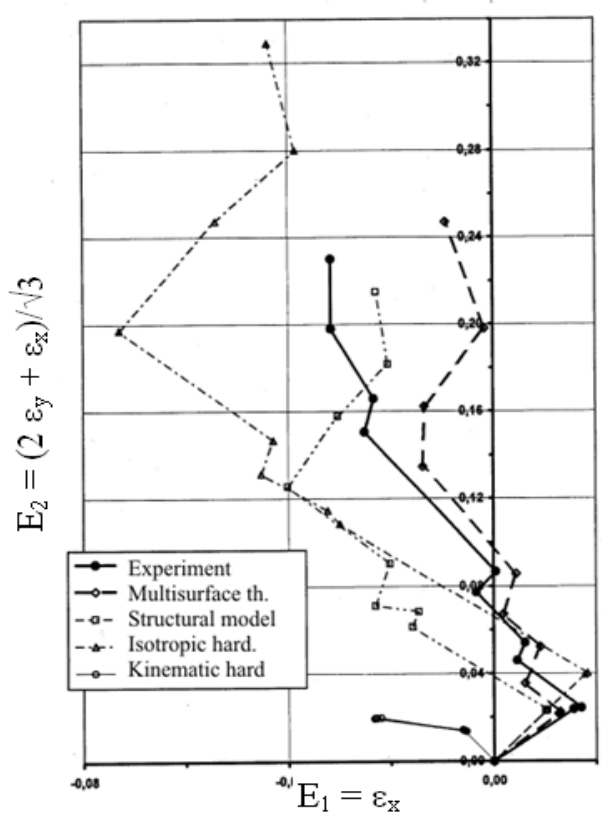

b)

Fig. 2. Comparison of different model predictions with experimental results for thin-walled tubes (steel $1 \mathrm{X} 18 \mathrm{H} 10 \mathrm{~T}$ ) under complex cyclic non-proportional loading (Izotov et al. 2001).

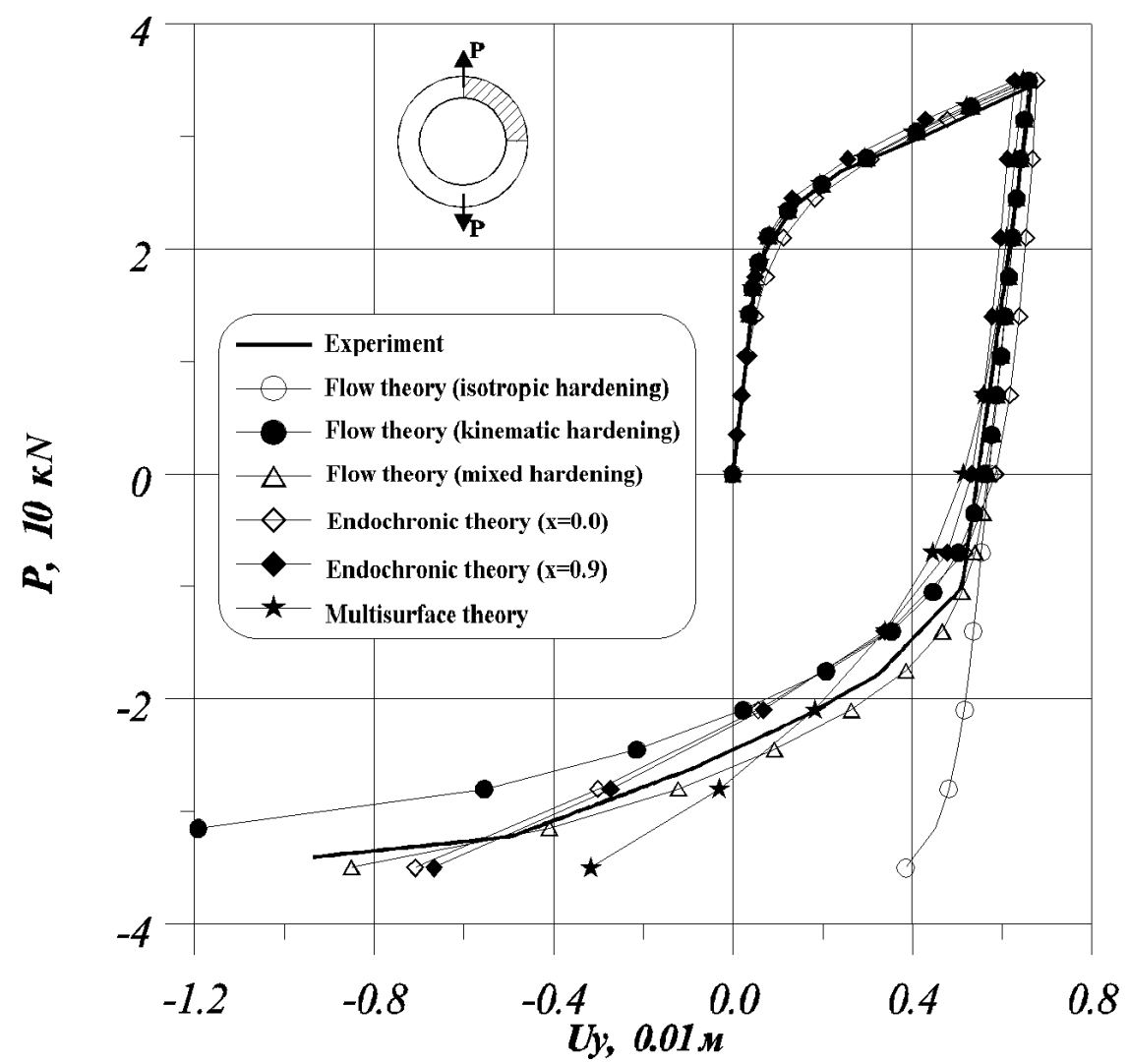

Fig. 3. Comparison of different model predictions with experimental results for circular ring under axial tension-compression (Semenov 1996). 
The multilevel finite element computations have been carried out for the fastening knot (see Fig. 4b), which is a part of the vapor producing plant (see Fig. 4a). The hysteresis curve, corresponding to tenth loading cycle, is shown in Fig. 4b. The results of simulations obtained at the level $\mathbf{E}$ (the fastening knot only analysis) improve the results corresponding to level $\mathbf{B}$ (the vapor producing plant analysis). The relative difference between predictions of different material models at the level $\mathbf{E}$ exceeds $50 \%$, while the difference at the level $\mathbf{B}$ is less than $10 \%$.

The levels $\mathbf{P}$ and $\mathbf{E}$ with the possibility of extensive zones of plasticity require the application of more complex variants of plasticity theory, which should be adequate to the complexity of loading process. Usage of more simple models is sufficient at the $\mathbf{B}$ level of the finite element simulation, when the local zones of plasticity are constrained by the extensive elastic domain.
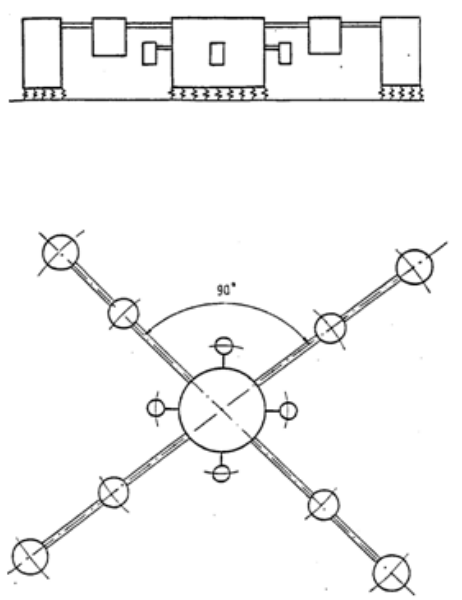

a)

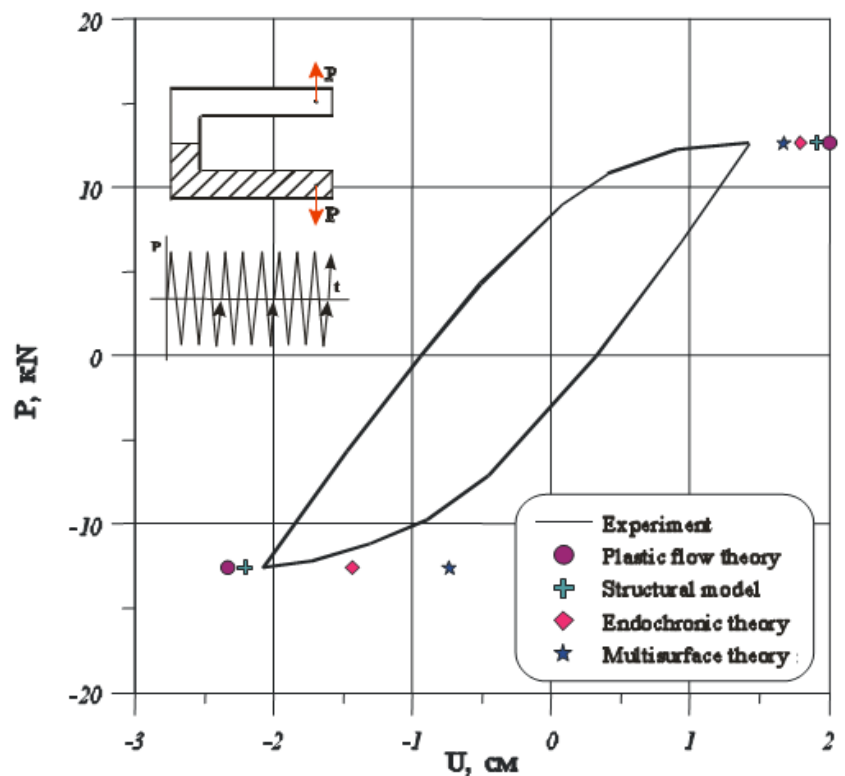

b)

Fig. 4. The vapor producing plant (a) and its part - the fastening knot (b) under cyclic loading (Melnikov, Semenov 1995).

\section{Conclusions}

A strategy of the automatic selection of an appropriate elastic-plastic material model during computation has been proposed within the framework of the iterative multimodel numerical analysis of complex structures. Certainly, the method does not claim to be fully completed now. The final purpose is a creation of the fully automatic system for the selection of adequate material models designed to ensure correct computations of the strain-stress state under complex variable nonproportional loading conditions with help of modern finite elements systems. The using the multimodel method can increase the effectiveness and reliability of computations, especially in the case of structure analysis under complex loading programs. We invite all authors of theories describing elasto-plastic and visco-elasto-plastic behavior of materials to take part in a joint development of the multimodel method and expansion of the material library.

\section{References}

Benallal, A., Cailletaud, G., Chaboche, J. L., Marquis, D., Nouailhas, D. and Rousset, M. 1989. Description and modelling of non-proportional effects in cyclic plasticity, in M.W. Brown and K.J. Miller (Eds.). Biaxial and Multiaxial
Fatigue. EGF 3. Mechanical Engineering Publications, London, 107-129.

Benin, A.V., Semenov, A.S., Semenov S.G., Melnikov, B.E. 2013. Simulation of pulling reinforcing bar from concrete. Part 1. Models with account of discontinuity. Magazine of Civil Engineering. 5(40): (in press).

Getsov, L.B., Melnikov, B.E., Semenov, A.S. 2002. Criteria of choice of thermo-visco-plastic models in stress-strain state analysis of structures, in Proc. of the 1st conf. of users of programs from CAD-FEM GmbH, Moscow, 340-352.

Getsov, L.B., Rybnikov, A.I., Semenov, A.S. 2009. Thermal fatigue strength of heat-resistant alloys. Thermal Engineering. 56 (5), pp. 412-420. http://dx.doi.org/10.1134/S0040601509050103

FE program PANTOCRATOR. 2013. Available from: $<$ http://www.pantocrator.narod.ru/>. [1 July 2013].

Izotov, I.N., Kuznetsov, N.P., Melnikov, B.E., Mityukov, A.G., Musienko, A.Y., Semenov, A.S. 2001. Modification of the multisurface theory of plasticity with one surface. Comparison with experimental data, Proceedings of SPIE - The International Society for Optical Engineering, 4348: p. 390-397.

Jirasek, M., Bazant, Z.P. Inelastic analysis of structures. 2001.

Kadashevich, Yu.I., Pomytkin, S.P. 2013. Endochronic theory of plasticity generalizing Sanders-Klyushnikov theory, Magazine of Civil Engineering 1(36): 82-86.

http://dx.doi.org/10.5862/MCE.36.10 
Kalinin A.L. 2013. Application of modified yield criteria for calculation of safe pressures on the subgrade soil]. Magazine of Civil Engineering. No.4(39): pp. 35-45 (in Russian) http://dx.doi.org/10.5862/MCE.39.4

Khan, A.S., Huang, S. 1995. Continuum theory of plasticity. Wiley \& Sons Australia, Ltd. 1995. 440 p.

Kolarov, D., Baltov, A., Boncheva, N. 1979. Mechanika plasticheskikh sred. Mir. 1979. 302p. (in Russian).

Lemaitre, J. (ed.) 2001, Handbook of Materials Behavior Models. Ed. Academic Press, 2001, 900 p.

Melnikov, B.E., Semenov, A.S. 1995. Strategy of multimodel analysis of elastic-plastic stress-strain state, in Proc. Int. Conf. on Comp. in Civil and Build. Eng. Berlin, 1073-1079.

Melnikov, B.E., Semenov, A.S. Multimodel analysis as strategy of reliable description of elastic-plastic deformation processes, in D.R.J. Owen, et al. (Eds.). Proc. IV Int. Conf. on Computational Plasticity. Fundamentals and Applications (COMPLAS IV). Pineridge Press. 1995. 181-189.

Melnikov, B.E., Semenov, A.S. 1996. Creation and application of hierarchical sequence of material models for numerical analysis of elasto-plastic structures. ZAMM. Zeitschrift fur Angewandte Mathematik und Mechanik, 76 (SUPPL. 2), pp. 615-616.

Novozhilov, V.V. 1964. O slozhnom nagruzhenii i pezspektivakh fenomenologicheskogo podkhoda $\mathrm{k}$ issledovaniyu mikronaprazhenii, Prikl. Mat. i Mech. 28(3): 393-400. (in Russian).

Ottosen, N.S. 1979. Nonlinear kinematic hardening under NonProportional Loading. Risø National Laboratory, DK-4000 Roskilde, Denmark.

Prager, W. 1949. Recent developments in the mathematical theory of plasticity, Journal of Applied Physics, 20(3): 235-241. http://dx.doi.org/10.1063/1.1698348

Semenov, A.S., Melnikov, B.E. 1998. Multimodel analysis of the elasto-plastic and elasto-visco-plastic deformation processes in materials and structures, in Proc. Int. Conf. Low Cycle Fatigue and Elasto-Plastic Behavior of Materials, Garmisch-Partenkirchen, 659-664.
Semenov, A.S. 1996. Improvement of the research methods of thermo-elasto-plastic deformation processes on the base of multimodel analysis and the exact integration of constitutive equations. Ph.D. thesis. St.-Petersburg. 194 p.

Semenov, A.S. 2003. PANTOCRATOR - finite-element program specialized on the solution of non-linear problems of solid body mechanics, in Proc. of 5th Int. Conf. Sci. and Eng. Problems of Reliability and Service Life of Structures and methods of their decision, St.-Petersburg, 466-480.

Semenov, A.S., Melnikov, B.E., Gorokhov, M.Y. 2005. About the causes of cyclical instability at computations of large elasto-plastic strains. Proceedings of SPIE - The International Society for Optical Engineering, 5831, art. no. 25, pp. 167-173.

Semenov, A.S., Melnikov, B.E., Gorokhov, M.Yu., Ulbricht, V. 2007. Prevention of cyclic instability at the modeling of elasto-plastic deformation at large strains under proportional and non-proportional loading. Proceedings of SPIE - The International Society for Optical Engineering, 6597, art. no. 659710 .

Szabo, L. 1984. Comparison of some plasticity theories by means of the finite element method. Periodica Polytechnica, Mechanical Engineering, 28(2-3): 153-161.

Tanaka, E., Murakami, S., Ooka, M. 1985. Effects of strain path shapes on non-proportional cyclic plasticity, J. Mech. Phys. Solids. 33: 559-575. http://dx.doi.org/10.1016/00225096(85)90003-1

Vasin, R.A. 1990. Constitutive equations of plasticity theory, Adv. Sci. and Eng. Solid body mechanics. 21: 3-75.

Zyczkowski, M. 1981. Combined Loadings in the Theory of Plasticity. PWN-Polish Scientific Publishers, Warszawa. $714 \mathrm{p}$.

\section{Acknowledgements}

The reported study was partially supported by RFBR, research project 12-08-00-943a. 